\title{
Anwältin heute
}

\author{
Workshop anlässlich des djb-Bundeskongresses am 23. September 2007 in Erfurt \\ Annette Peteranderl, Rechtsanwältin, Saarbrücken
}

Am letzten Vormittag des djb-Kongresses in Erfurt nahm ich an dem Workshop „Anwältin heute“ teil, der von den Rechtsanwältinnen Renate Maltry, Vizepräsidentin des djb, und Dr. Angelika Nake, Vorsitzende der Kommission Zivilrecht, angeboten wurde.

Diskussionspunkte waren u.a. die Gefahr von Gewalt bei der Wahrnehmung von Mandaten und die möglichen Schutzmaßnahmen von Seiten der Gerichte. Anlass war der bekannte Fall einer Kollegin in Berlin. Da wir in der Runde keine repräsentativen Erfahrungen hatten, wurde angeregt, eine Mitgliederumfrage durchzuführen.
Ein weiterer Punkt war die Frage: Was ist eine zertifizierte Kanzlei und wie wird man das?

Wenn man sich im alltäglichen „Arbeitstreiben " befindet, ist es schwierig, alte Strukturen und Arbeitsweisen zu ändern; dazu zwingt das Zertifizierungsverfahren. Scheut man die damit verbundenen Kosten, so ist es dennoch sinnvoll, sich regelmäßig die Zeit zu nehmen, um mit den Kolleginnen und Kollegen über die Ziele der Kanzlei, die Personalstruktur, die Arbeitsabläufe etc. zu diskutieren. Als selbstständige Anwältin ist man nie „nur“ Juristin, sondern immer auch Unternehmerin, die betriebswirtschaftlich denken muss und Verantwortung für ihre Mitarbeiter trägt. Das Gespräch mit den zwei erfahrenen Kolleginnen empfand ich als sehr wertvoll. In angenehm kleiner Runde konnte auf Fragen intensiv eingegangen werden.

Herzlichen Dank, Frau Maltry und Frau Dr. Nake!

\section{Herzlicher Dank an die Patinnen!}

\author{
Vivien Weidauer | Schriftführerin des LV Berlin, Assessorin in Berlin
}

Traditionell spendet zu jedem Kongress eine ganze Reihe von djb-Frauen einen bestimmten Betrag, um anderen, meist jüngeren Kolleginnen die Kongressteilnahme zu ermöglichen oder diese durch eine finanzielle Unterstützung zu erleichtern.
Auch für den 37. Kongress in Erfurt haben Patinnen großzügig gespendet. Auch im Namen meiner Kolleginnen unter den jüngeren Juristinnen möchte ich allen Patinnen danken! Ich selbst konnte auf diesem Weg an dem Kongress teilnehmen und habe einen Teil der Fahrtkosten und Übernachtungskosten erstattet bekommen. Ohne die Hilfe der Patinnen hätte ich mir den Kongress samt 3-tägigem Aufenthalt in Erfurt nicht ohne weiteres leisten können.

\section{Ausschreibung}

Gründung einer Arbeitsgruppe „Juristenausbildung“

Im Zusammenhang mit der Diskussion um eine Reform der Juristenausbildung möchte der Bundesvorstand eine Arbeitsgruppe damit beauftragen, die im Jahr 1998 vom djb hierzu formulierten Thesen weiterzuentwickeln. Bei der Erstellung eines Reformvorschlags sollten insbesondere der Bologna-Prozess und die damit verbundene Anpassung des Jurastudiums auf Bachelor- und Masterstudiengänge Berücksichtigung finden.

Kolleginnen, die an einer Mitarbeit interessiert sind, werden gebeten, sich bis Ende Juni bei der djb-Bundesgeschäftsstelle, Anklamer Straße 38, 10115 Berlin, zu melden. 